\title{
Migration of a sphere in tube flow
}

\author{
By B. H. YANG ${ }^{1}$, J. WANG ${ }^{1}$, D. D. JOSEPH ${ }^{1}$, H. H. HU ${ }^{2}$, \\ T.-W. PA N ${ }^{3}$ AND R. GLOWINSKI ${ }^{3}$ \\ ${ }^{1}$ Department of Aerospace Engineering and Mechanics, University of Minnesota, \\ Minneapolis, MN 55455, USA \\ ${ }^{2}$ Department of Mechanical Engineering and Applied Mechanics, University of Pennsylvania, \\ Philadelphia, PA 19104, USA \\ ${ }^{3}$ Department of Mathematics, University of Houston, Houston, TX 77204, USA
}

(Received 30 March 2004 and in revised form 13 April 2005)

The cross-stream migration of a single neutrally buoyant rigid sphere in tube flow is simulated by two packages, one (ALE) based on a moving and adaptive grid and another (DLM) using distributed Lagrange multipliers on a fixed grid. The two packages give results in good agreement with each other and with experiments. A lift law $L=C U_{s}\left(\Omega_{s}-\Omega_{s e}\right)$ analogous to $L=\rho U \Gamma$ which was proposed and validated in two dimensions is validated in three dimensions here; $C$ is a constant depending on material and geometric parameters, $U_{s}$ is the slip velocity and it is positive, $\Omega_{s}$ is the slip angular velocity and $\Omega_{s e}$ is the slip angular velocity when the sphere is in equilibrium at the Segré-Silberberg radius. The slip angular velocity discrepancy $\Omega_{s}-\Omega_{s e}$ is the circulation for the free particle and it changes sign with the lift. A method of constrained simulation is used to generate data which is processed for correlation formulas for the lift force, slip velocity, and equilibrium position. Our formulae predict the change of sign of the lift force which is necessary in the Segré-Silberberg effect. Our correlation formula is compared with analytical lift formulae in the literature and with the results of two-dimensional simulations. Our work establishes a general procedure for obtaining correlation formulae from numerical experiments. This procedure forms a link between numerical simulation and engineering practice.

\section{Introduction}

Migration and equilibrium of solid particles in shear flows have always been of great interest. Segré \& Silberberg $(1961,1962)$ studied the migration of dilute suspensions of neutrally buoyant spheres in tube flows and found the particles migrate away from both the wall and the centreline and accumulate at a radial position of about 0.6 times the tube radius. This remarkable Segré-Silberberg effect has been verified by many experimental works on the same kind of problem. For example, Goldsmith \& Mason (1962) observed that a rigid particle stayed at the initial radial position at very small Reynolds numbers and migrated to intermediate positions at finite Reynolds numbers. Karnis, Goldsmith \& Mason (1966) reported that neutrally buoyant particles stabilized midway between the centreline and the wall, closer to the wall for larger flow rates and closer to the centre for larger particles.

In an attempt to explain the Segré-Silberberg effect, different analytical expressions for the lift force which causes the particle to migrate transversely were obtained in the literature (Rubinow \& Keller 1961; Bretherton 1962; Saffman 1965, 1968; 
McLaughlin 1991; Auton 1987 among others). These analytical expressions are based on perturbing Stokes flow with inertia or on perturbing potential flow with a little vorticity. They are explicit and valuable; however, the restrictions on the perturbation analyses make it difficult to apply these expressions to practical problems where the Reynolds number is finite and viscosity is important.

Schonberg \& Hinch (1989) analysed the lift on a neutrally buoyant small sphere in a plane Poiseuille flow, with the channel Reynolds number $R_{c}=U_{m}^{\prime} l / v$ less than approximately 100, using matched asymptotic methods. Here, $U_{m}^{\prime}$ is the maximum velocity of a channel flow, and $l$ is the channel width. The same problem for neutrally buoyant and non-neutrally buoyant small spheres has been studied by Asmolov (1999). He considered the Reynolds number based on the particle size to be asymptotically small, while the channel Reynolds number is finite. He computed the lift force on a sphere as a function of the distance from the wall to the sphere centre for flows with $R_{c}$ up to 3000 ; the results show that the equilibrium position moves towards the wall as $R_{c}$ increases. When the particle Reynolds number is small, the disturbance flow due to the particle is governed by creeping-flow equations to leading order; one can compute the transverse component of the velocity at the particle centre and use the Stokes drag law to calculate the lift. The analysis takes the effect of inertia $(\boldsymbol{u} \cdot \nabla) \boldsymbol{u}$ into account only in an Oseen linear system. The analysis is heavy and explicit formulae for the lift are not obtained.

Feng, Hu \& Joseph (1994) performed numerical simulations of the motion of a two-dimensional circular particle in Couette and Poiseuille flows. Patankar et al. (2001a) and Joseph \& Ocando (2002) simulated the motion of a two-dimensional circular particle in plane Poiseuille flows perpendicular to gravity in Newtonian and viscoelastic fluids. They showed that multiple equilibrium states exist for particles with intermediate densities; these equilibrium states can be stable or unstable.

Relative motions between the fluid and the particle, which may be characterized by slip velocities, are essential to understand the lift force on the particle. We use $U_{p}$ and $\Omega_{p}$ to denote the translational and angular velocities of the particle at steady state and the slip velocities are defined as:

$$
\begin{gathered}
U_{s}=U_{f}-U_{p}, \text { the slip velocity, } \\
\Omega_{s}=\Omega_{p}-\Omega_{f}=\Omega_{p}+\dot{\gamma} / 2, \text { the slip angular velocity },
\end{gathered}
$$

where $U_{f}$ and $\dot{\gamma}$ are the fluid velocity and local shear rate evaluated at the location of the particle centre in the undisturbed flow. Joseph \& Ocando (2002) found that the discrepancy $\Omega_{s}-\Omega_{s e}$, where $\Omega_{s e}$ is the slip angular velocity at equilibrium, changes sign across the equilibrium position just as the lift force does. Thus, this discrepancy can be used to account for the migration toward the intermediate equilibrium position from the centreline and the wall (the Segré-Silberberg effect).

Following Joseph \& Ocando's analysis, Wang \& Joseph (2003) constructed correlations for the lift force by analogy with the classical lift formula $L=\rho U \Gamma$ of aerodynamics; they showed that the proper analogues of $U$ and $\Gamma$ were $U_{s}$ and $\Omega_{s}-\Omega_{s e}$. Their correlations apply to a freely rotating two-dimensional circular particle without accelerations in a plane Poiseuille flow. They also demonstrated that the correlations for lift force could be made completely explicit provided that the correlations relating $U_{s}$ and $\Omega_{s}$ to prescribed parameters were obtained.

The results from two-dimensional simulations presented by Feng et al. (1994), Patankar et al. (2001a), Joseph \& Ocando (2002) and Wang \& Joseph (2003) are 
difficult to compare with experimental results because the majority of the experiments are for spherical particles in circular tube flows. The lift force correlations by Wang \& Joseph are for circular particles and cannot be rigorously compared to analytical lift expressions for spheres. All the above mentioned authors used a two-dimensional finite-element scheme based on unstructured body-fitted moving grids first developed by $\mathrm{Hu}$, Joseph \& Crochet (1992). Hu \& Zhu extended the two-dimensional scheme to three-dimensional and performed simulations of the migration of spheres in tube Poiseuille flows (see Zhu 2000). We call this scheme the ALE code because an arbitrary Lagrangian-Eulerian moving mesh technique has been adopted to deal with the motion of the particles. The three-dimensional ALE scheme is used in the current work to study the lift force on a neutrally buoyant sphere in tube Poiseuille flow.

Another approach to simulate the solid-liquid flow initiated by us is based on the principle of embedded or fictitious domains. In this approach, the Navier-Stokes equations are solved everywhere, including inside the particles. The flow inside the particles is forced to be a rigid-body motion by a distribution of Lagrange multipliers thus we call this scheme the DLM code. Detailed descriptions of the DLM method can be found in Glowinski, Pan \& Periaux (1998), Glowinski et al. (1999a, b, 2001), Singh et al. (2000) and Glowinski (2003). The two quite different schemes, the ALE code and the DLM code, are both employed to simulate the motion of a neutrally buoyant sphere in tube Poiseuille flow in the current work. We shall show that the results of the two codes are in good agreement, which provides strong support for the credibility of our numerical simulation.

The main goal of this work is to extend the correlation of the lift force in terms of $U_{s}$ and the discrepancy $\Omega_{s}-\Omega_{s e}$ to three-dimensional cases, which would give a more convincing explanation for the Segré-Silberberg effect. The lift force correlations represent efforts to draw explicit formulae from numerical simulation data. There is no reason why 'empirical' formulae of the type used in engineering which correlate experimental data cannot be generated from the data produced by numerical simulations. Correlations have already been obtained for single particle lift (Patankar et al. 2001a; Wang \& Joseph 2003) and for the bed expansion of many particles (Choi \& Joseph 2001; Patankar et al. 2001b) by processing numerical data. Our works have established general rules for the interrogation of data from numerical simulation to be used in developing models for complex fluid dynamics problems such as the fluidization by lift or drag.

\section{Governing equations and dimensionless parameters}

The governing equations for the incompressible Newtonian fluid are

$$
\nabla \cdot \boldsymbol{u}=0, \rho_{f}\left(\frac{\partial \boldsymbol{u}}{\partial t}+(\boldsymbol{u} \cdot \nabla) \boldsymbol{u}\right)=-\nabla P+\rho_{f} \boldsymbol{g}+\mu \nabla^{2} \boldsymbol{u},
$$

where $\boldsymbol{u}(\boldsymbol{x}, t)$ is the fluid velocity, $\rho_{f}$ is the fluid density, $P(\boldsymbol{x}, t)$ is the pressure, $\boldsymbol{g}$ is the acceleration due to gravity, and $\mu$ is the fluid viscosity. We can absorb the gravity term in the Navier-Stokes equations into the pressure by decomposing the pressure as

$$
P=p+\rho_{f} \boldsymbol{g} \cdot \boldsymbol{x}
$$


The equations of motion of the solid particles in a general three-dimensional case are

$$
\begin{gathered}
m \frac{\mathrm{d} \boldsymbol{U}_{p}}{\mathrm{~d} t}=m \boldsymbol{g}+\int[-P \mathbf{1}+\boldsymbol{\tau}] \cdot \boldsymbol{n} \mathrm{d} \Gamma, \\
\frac{\mathrm{d}\left(\boldsymbol{I} \cdot \boldsymbol{\Omega}_{p}\right)}{\mathrm{d} t}=\int\left(\boldsymbol{x}-\boldsymbol{X}_{p}\right) \times([-P \mathbf{1}+\boldsymbol{\tau}] \cdot \boldsymbol{n}) \mathrm{d} \Gamma,
\end{gathered}
$$

where $m$ is the mass of the particle, $\boldsymbol{U}_{p}$ is the translational velocity, $\boldsymbol{\Omega}_{p}$ is the angular velocity, $\mathbf{I}$ is the unit tensor, $\boldsymbol{I}$ is the moment of inertia tensor and $\boldsymbol{X}_{p}$ is the coordinate of the centre of mass of the particle. We consider a solid sphere with a radius $a$ and $m=\rho_{p} 4 \pi a^{3} / 3, \boldsymbol{I}=\operatorname{diag}\left(2 m a^{2} / 5\right)$. The no-slip condition is imposed on the particle boundaries

$$
\boldsymbol{u}=\boldsymbol{U}_{p}+\boldsymbol{\Omega}_{p} \times\left(\boldsymbol{x}-\boldsymbol{X}_{p}\right)
$$

and on the tube wall $\boldsymbol{u}=0$. The velocity profile at the inlet of the tube is prescribed by

$$
u=U_{m}\left(1-r^{2} / R^{2}\right)
$$

where $U_{m}$ is the maximum velocity at the centreline of the tube; $r$ is the radial position and $R$ is the radius of the tube. The traction-free boundary condition is imposed at the outlet of the tube:

$$
f_{n} \equiv \boldsymbol{n} \cdot \boldsymbol{T} \cdot \boldsymbol{n}=0 ; \quad u_{y}=0 ; \quad u_{z}=0,
$$

where $\boldsymbol{T}=-P \mathbf{1}+\boldsymbol{\tau}$ is the total stress tensor and $\boldsymbol{n}$ is the norm at the outlet of the tube.

The undisturbed tube Poiseuille flow without particles is given by (2.5). The shear rate at the wall $(r=R)$ is given by $\dot{\gamma}_{w}=2 U_{m} / R$. To non-dimensionalize the governing equations, we use the velocity $V=2 a \dot{\gamma}_{w}$ as the characteristic velocity, $2 a$ for length, $2 a / V$ for time, $\mu V / 2 a$ for stress and pressure, and $\dot{\gamma}_{w}$ for angular velocity. The dimensionless equations are (we use the same symbols for dimensionless variables)

$$
\begin{gathered}
\nabla \cdot \boldsymbol{u}=0, \\
\operatorname{Re}\left(\frac{\partial \boldsymbol{u}}{\partial t}+(\boldsymbol{u} \cdot \nabla) \boldsymbol{u}\right)=-\nabla p+\nabla^{2} \boldsymbol{u}, \\
\frac{\rho_{p}}{\rho_{f}} \operatorname{Re} \frac{\mathrm{d} \boldsymbol{U}_{p}}{\mathrm{~d} t}=G \boldsymbol{e}_{g}+\frac{6}{\pi} \int[-p \mathbf{1}+\boldsymbol{\tau}] \cdot \boldsymbol{n} \mathrm{d} \Gamma, \\
\frac{\rho_{p}}{\rho_{f}} \operatorname{Re} \frac{\mathrm{d} \boldsymbol{\Omega}_{p}}{\mathrm{~d} t}=\frac{60}{\pi} \int\left(\boldsymbol{x}-\boldsymbol{X}_{p}\right) \times([-p \mathbf{1}+\boldsymbol{\tau}] \cdot \boldsymbol{n}) \mathrm{d} \Gamma,
\end{gathered}
$$

where $\boldsymbol{e}_{g}$ is the unit vector in the direction of the gravity. The dimensionless parameters are

$$
\begin{gathered}
R e=\frac{\rho_{f} V(2 a)}{\mu}=\frac{\rho_{f} \dot{\gamma}_{w}(2 a)^{2}}{\mu}=\frac{8 a^{2} \rho_{f} U_{m}}{\mu R}, \text { the Reynolds number, } \\
G=\frac{\left(\rho_{p}-\rho_{f}\right) g(2 a)^{2}}{\mu V}, \text { the gravity number, } \\
\rho_{p} / \rho_{f}, \text { the density ratio. }
\end{gathered}
$$

It is convenient to carry out the analysis of correlations in terms of dimensionless forms of correlating parameters. The ratio of the sphere radius $a$ to pipe radius $R$ 


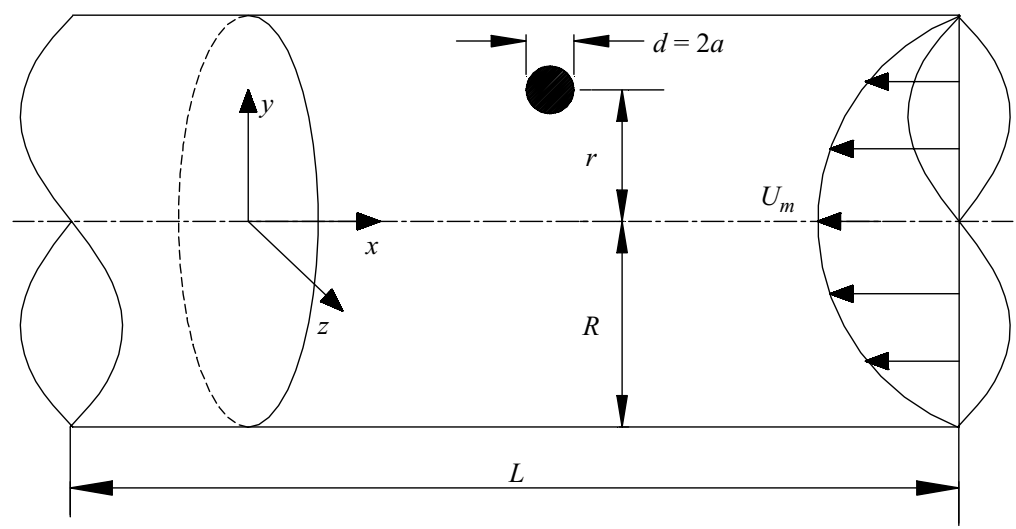

FIGURE 1. Sketch for the problem of a rigid sphere in a tube Poiseuille flow. The sphere is neutrally buoyant and the orientation of the gravity is irrelevant.

and the dimensionless radial position $\bar{r}$ are defined by

$$
\bar{a}=a / R, \quad \bar{r}=r / R .
$$

The dimensionless lift is given by

$$
\bar{L}=\frac{6 \rho_{f} L}{\pi \mu^{2}} .
$$

The flow quantities $U_{m}, U_{s}, \Omega_{s}, \Omega_{s e}$ are expressed in the form of Reynolds numbers. A flow Reynolds number is given by

$$
\bar{U}_{m}=\frac{\rho_{f} U_{m} R}{\mu}=\frac{R e}{8 \bar{a}^{2}} .
$$

Slip velocity Reynolds numbers are defined as

$$
\left.\begin{array}{l}
\bar{U}_{s}=\rho_{f} U_{s}(2 a) / \mu, \\
\bar{\Omega}_{s}=\rho_{f} \Omega_{s}(2 a)^{2} / \mu, \\
\bar{\Omega}_{s e}=\rho_{f} \Omega_{s e}(2 a)^{2} / \mu .
\end{array}\right\}
$$

A dimensionless form of the product $U_{s}\left(\Omega_{s}-\Omega_{s e}\right)$ which enters into our lift law is given as the product

$$
\bar{U}_{s}\left(\bar{\Omega}_{s}-\bar{\Omega}_{s e}\right) .
$$

Note that the flow is in the negative $x$-direction in our simulation (see figure 1 ). The symbol $U_{m}$ in (2.10) and (2.15) should be understood as the magnitude of the fluid velocity at the tube centreline. Similarly, we use the magnitude of $U_{f}$ and $U_{p}$ to calculate the slip velocity $U_{s}$ defined in (1.1).

We shall focus on the steady-state flow of a neutrally buoyant sphere, in which the left-hand side of (2.9) and the term $G \boldsymbol{e}_{g}$ in (2.9) vanish. Thus, $R e$ and $\bar{a}$ are the two parameters in play.

\section{Comparison of results}

We study the behaviour of a neutrally buoyant sphere suspended in tube Poiseuille flows. A comparison of the numerical results using the ALE code with the experiments by Karnis et al. (1966) was presented by Zhu (2000). Karnis et al. performed a large 


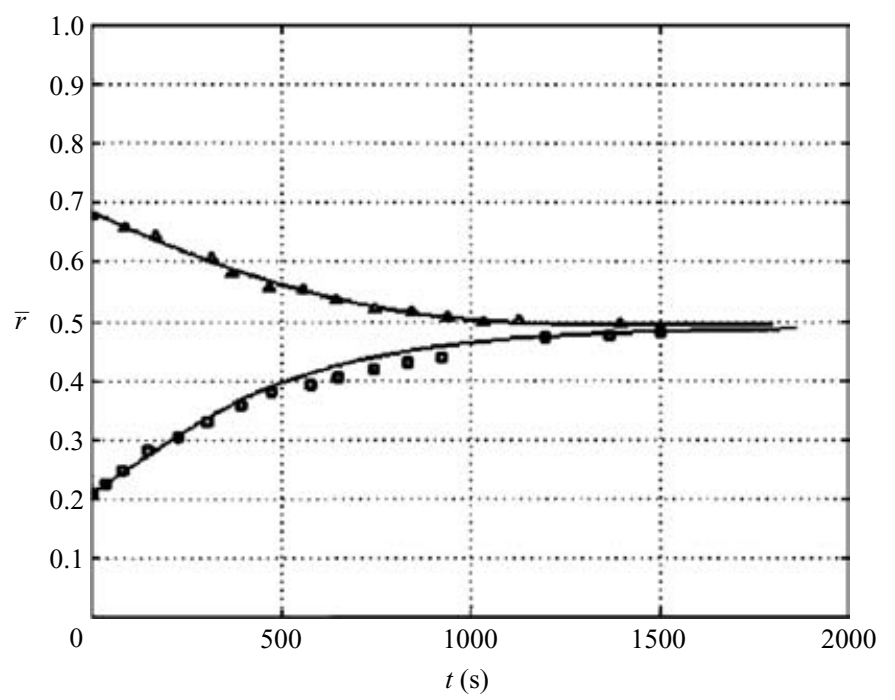

FIGURE 2. Comparison of migration trajectories of a neutrally buoyant sphere calculated numerically by Zhu (2000) (-) with those measured in the experiments of Karnis et al. (1966) $(\bigcirc, \bar{r}=0.21 ; \Delta, 0.68)$. The fluid properties are $\rho_{f}=1.05 \mathrm{~g} \mathrm{~cm}^{-3}$ and $\mu=1.2$ poises, the flow rate is $Q=7.11 \times 10^{-2} \mathrm{~cm}^{3} \mathrm{~s}^{-1}$, the tube diameter is $D=0.4 \mathrm{~cm}$, and the sphere diameter is $d=0.122 \mathrm{~cm}$.

number of experiments on the migration of spheres, rods and disks in a Poiseuille flow in a capillary tube. Zhu (2000) compared the trajectories of spheres released at two radial positions $\bar{r}=0.21$ and 0.68 to the trajectories measured by Karnis et al. Figure 2 shows that the numerical results are in excellent agreement with the experimental ones.

An equilibrium for a free neutrally buoyant particle is achieved when the particle migrates to a radial position $\bar{r}_{e}$ of steady rectilinear motion in which the acceleration and angular acceleration vanish and the hydrodynamic lift force is zero. We perform two types of simulation, unconstrained and constrained simulations, to find the equilibrium position. In unconstrained simulation, the particle moves freely until it reaches its equilibrium position, just as it would do in experiments. In constrained simulation, the particle is only allowed to move along a line parallel to the axis of the tube and rotate freely; its lateral migration is suppressed and radial position is fixed. When such a constrained motion reaches a steady state in which the particle accelerations vanish, a hydrodynamic lift force $L$ in the radial direction can be calculated. This lift force $L$ is a function of the radial position; the position where $L=0$ is the equilibrium position $\bar{r}_{e}$. In our constrained simulation, we place the sphere centre at $(y=r, z=0)$ (see figure 1), so that the lift force at steady state is in the $y$-direction, and the $z$-direction is the neutral direction. The only component of the particle translational velocity at steady state is $U_{x}$, and $\Omega_{z}$ is the only component of the angular velocity.

We compare the results of the unconstrained and constrained simulations obtained using the ALE code and the DLM code. In the ALE code, the typical mesh used in most of our simulations consists of $1.05 \times 10^{5}$ elements, $1.46 \times 10^{5}$ nodes and $1.9 \times 10^{4}$ vertices. The number of nodes for velocity is about $2.22 \times 10^{6}$ in the DLM code. We consider a case in which the radii of the particle and the tube are $0.375 \mathrm{~cm}$ and $2.5 \mathrm{~cm}$, respectively (the radius ratio $\bar{a}=0.15$ ), the density of the particle and the 


$\begin{array}{lccc} & \bar{r}_{e} & U_{x}\left(\mathrm{~cm} \mathrm{~s}^{-1}\right) & \Omega_{z}\left(\mathrm{~s}^{-1}\right) \\ \text { ALE } & 0.601 & 12.4 & 4.65 \\ \text { DLM } & 0.606 & 12.2 & 4.63\end{array}$

TABLE 1 . The equilibrium position $\bar{r}_{e}$ and particle velocities $U_{x}$ and $\Omega_{z}$ at equilibrium calculated from the unconstrained simulation. The results of the ALE and DLM codes are almost the same.

fluid is $1 \mathrm{~g} \mathrm{~cm}^{-3}$, the viscosity of the fluid is 1 poise, and $U_{m}=20 \mathrm{~cm} \mathrm{~s}^{-1}(R e=9)$. We compare the equilibrium position $\bar{r}_{e}$ and velocities $U_{x}$ and $\Omega_{z}$ at equilibrium calculated from the unconstrained simulation in table 1 . We compare the lift force $L(\bar{r})$, velocities $U_{x}(\bar{r})$ and $\Omega_{z}(\bar{r})$, calculated from the constrained simulation in figure 3 .

Figures $3(a)$ and $3(b)$ show that the particle translational and angular velocities obtained from the DLM and ALE codes are almost the same. The agreement of the lift forces from the two codes in figure $3(c)$ is not as good as the velocities. The lift force curve from the DLM code is not quite smooth since this method was intended to simulate the dynamics of the interaction of fluid and many particles and not to compute the hydrodynamic forces explicitly. Nevertheless, we regard the agreement in figure 3(c) acceptable, considering the challenging nature of the three-dimensional simulation. Because of mesh adaptivity, the ALE code is more suited to accurate computation of lift. We will use the data from the ALE code to construct the lift correlations in the following section.

Our two different codes give results in good agreement with each other and with experiments, which demonstrates that our numerical simulation is credible.

\section{Correlations from the numerical simulation}

Numerical experiments using constrained simulation provide us with the distribution of the lift force and particle velocity in the tube and the position and velocity of the particle at equilibrium. We develop correlations for these quantities in this section. The key correlation is for the lift force, which shows the dependence of the lift force on the slip angular velocity discrepancy $\Omega_{s}-\Omega_{s e}$. The lift force correlation predicts the change of sign of the lift force, which is necessary to explain the two-way migration in the Segré-Silberberg effect. The correlations for the equilibrium state of the particle are also of interest, because they may be used to predict the position and the velocities of the particle at equilibrium.

\subsection{Correlation for the lift force}

The steady-state values of the lift forces on a sphere at different radial positions computed in constrained simulation are plotted in figure 4 for a sphere with the radius ratio $\bar{a}=0.15$. The positive direction of the lift force is in the negative $\boldsymbol{e}_{r}$ direction. In other words, $\bar{L}$ is positive when pointing to the centreline and negative when pointing away from the centreline.

The equilibrium positions of a neutrally buoyant sphere are the points where $\bar{L}=0$. The stability of the equilibrium at a zero-lift point can be determined from the slope of the $\bar{L}$ vs. $\bar{r}$ curve. The centreline is on a negative-slope branch of the $\bar{L} v s . \bar{r}$ curve. When a particle is disturbed away from the centreline, the lift force is negative and drives the particle further away from the centreline. Therefore, the centreline is 

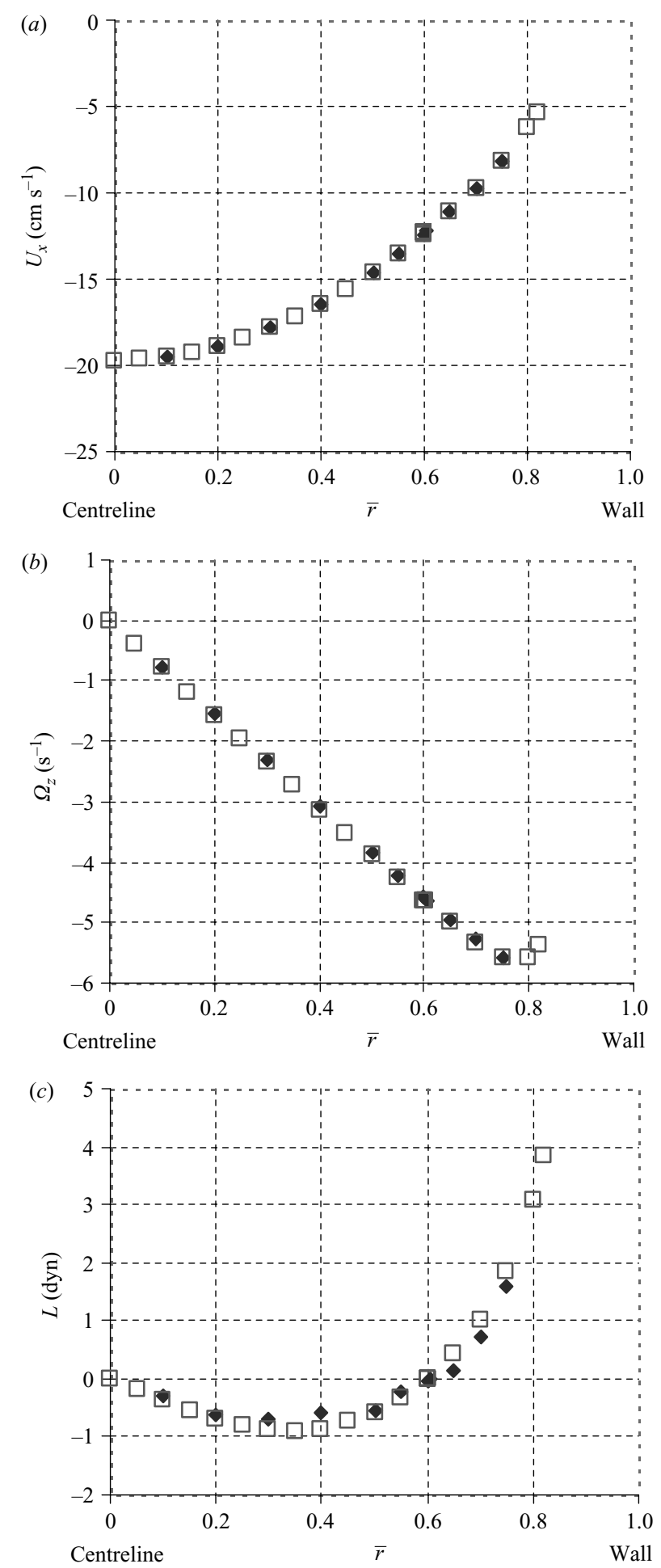

FiguRE 3. The steady-state values of the particle velocities $(a) U_{x}$ and $(b) \Omega_{z}$ and (c) the lift force $L$, from the constrained simulation at different radial positions. The $\square$, ALE and DLM results are in good agreement. 
(a)

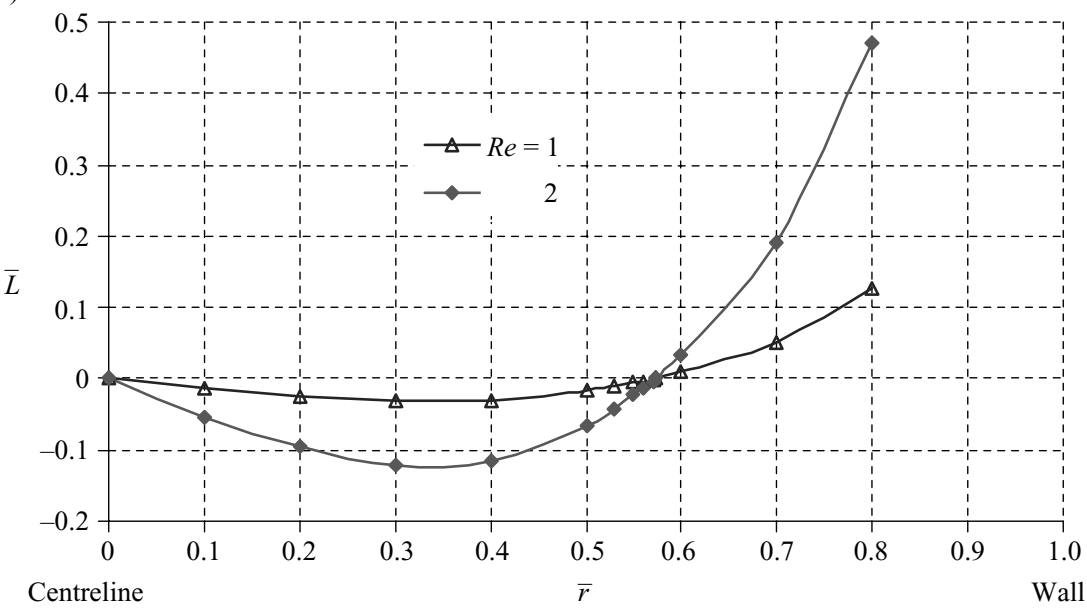

(b)

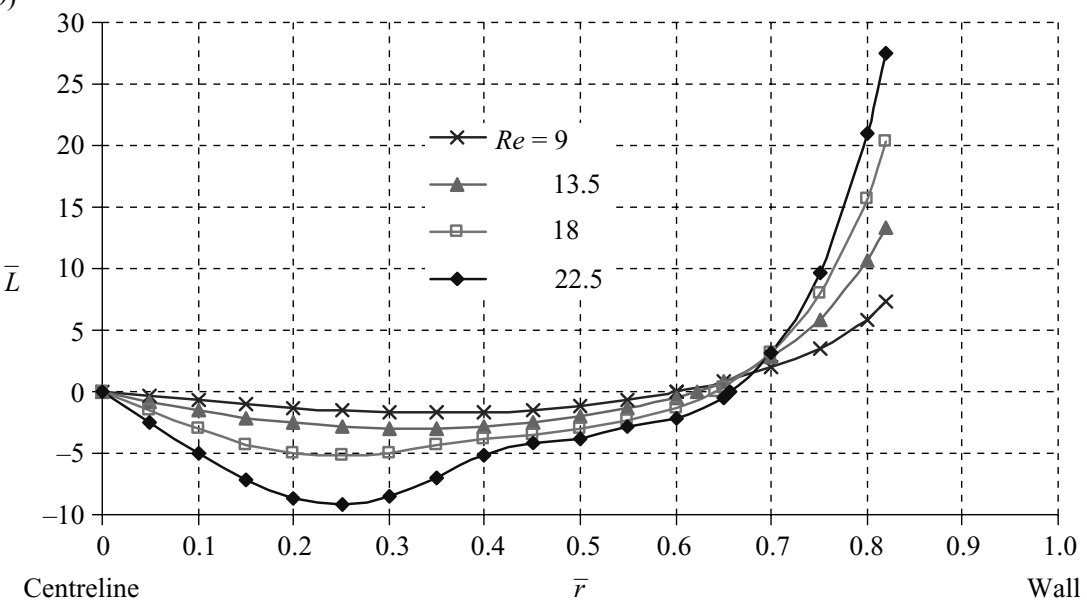

FiguRE 4. The dimensionless lift force $\bar{L}$ at different radial positions for a sphere with the radius ratio 0.15 . The two curves in $(a)$ correspond to $R e=1$ and 2 , and the four curves in (b) correspond to $R e=9,13.5,18$ and 22.5, respectively. The magnitude of the lift force increases with the Reynolds number.

an unstable equilibrium position. The other zero-lift point is between the centreline and the wall and it is on a positive-slope branch of the curve. When the particle is disturbed away from this point, the lift force tends to push the particle back. Thus the zero-lift point between the centreline and the wall is a stable equilibrium position. Figure 4 shows that this stable equilibrium position $\bar{r}_{e}$ moves towards the wall as the Reynolds number increases.

We seek expressions for the lift force in terms of the slip velocities. The slip velocity Reynolds numbers have been defined in (2.16). We plot $\bar{\Omega}_{s}-\bar{\Omega}_{s e}$ at different radial positions in figure 5 for a sphere with $\bar{a}=0.15$. Comparison of figures 4 and 5 shows that the discrepancy $\bar{\Omega}_{s}-\bar{\Omega}_{\text {se }}$ changes sign across the equilibrium position, as does the lift force $\bar{L}$.

If we fix the radius ratio $\bar{a}=0.15$ and continue to increase the Reynolds number, the distribution of the lift force as a function of the radial position becomes more 
(a)

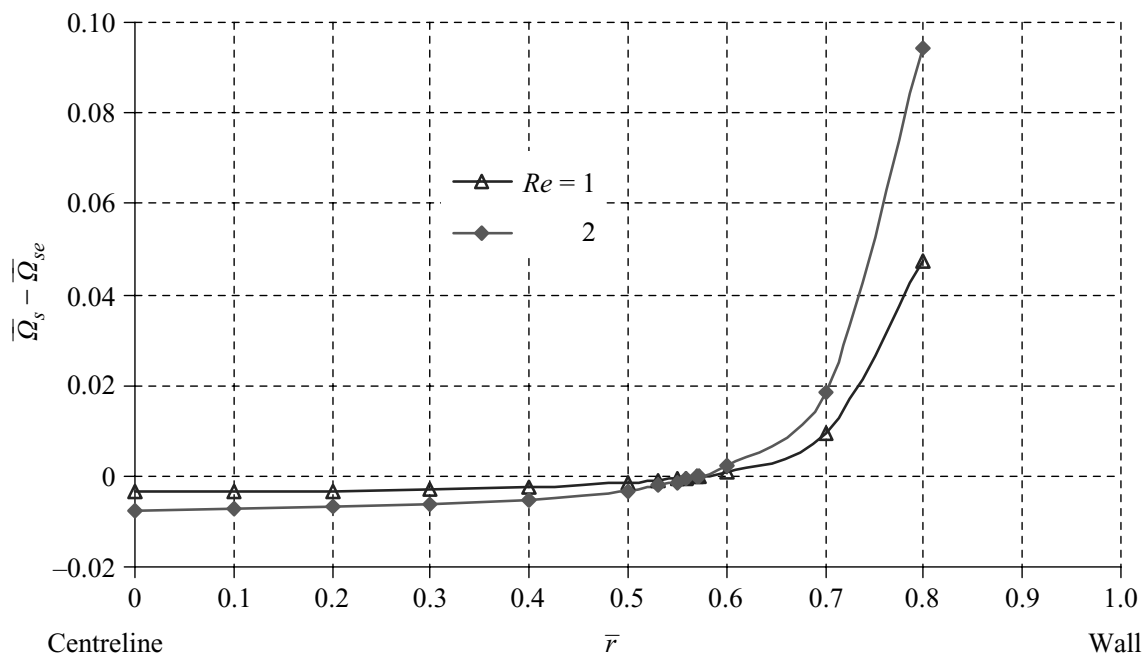

(b)

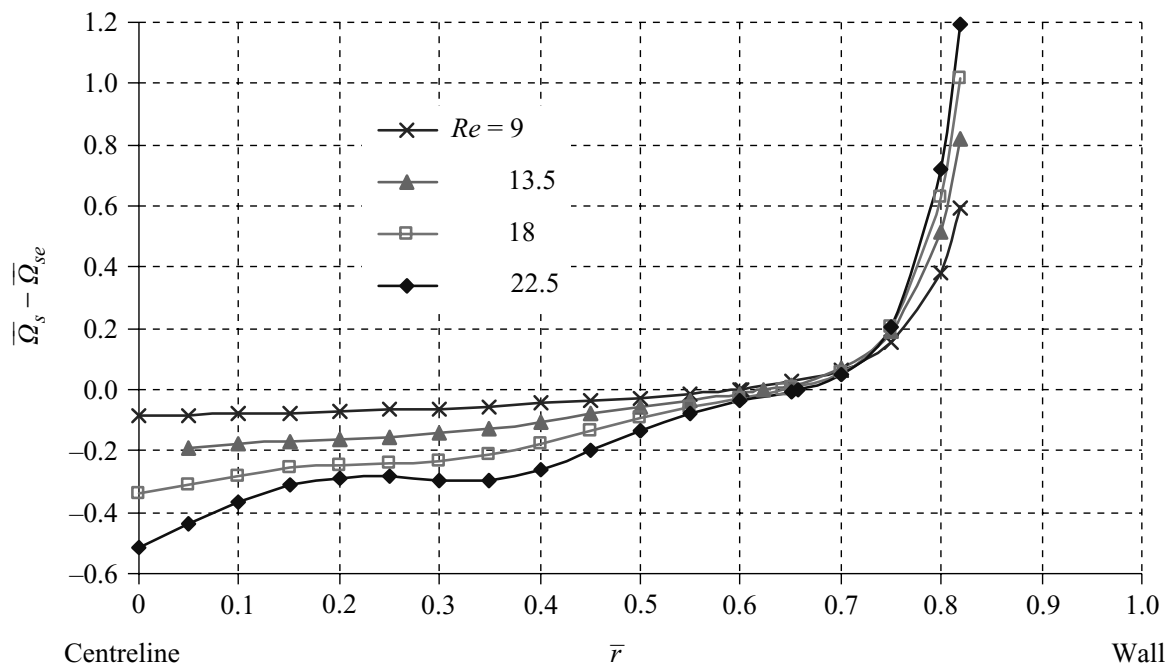

FIGURE 5. The dimensionless slip angular velocity discrepancy at different radial positions for a sphere with $\bar{a}=0.15$. The two curves in $(a)$ correspond to $R e=1$ and 2 , and the four curves in $(b)$ correspond to $R e=9,13.5,18$ and 22.5 , respectively.

complicated. We plot the $\bar{L}$ vs. $\bar{r}$ curves for $R e=27,36$ and 45 in figure 6 . There is a change of convexity in the curves and a local minimum of the lift force exists at approximately $\bar{r}=0.55$. On each of the curves, there exist two branches on which the slope of the curve is negative. The first negative-slope branch is at the centreline; the second negative-slope branch is in the middle between the centreline and the wall. The second negative-slope branch does not exist when the Reynolds number is small $(R e=1,2,9,13.5,18$ or 22.5$)$. The exact range of the second negative-slope branch varies with the Reynolds number; we may say that the range $0.5 \leqslant \bar{r} \leqslant 0.65$ covers the second negative-slope branches of the curves for $R e=27,36$ and 45 . We found that it was difficult for the constrained simulations in this range at high Reynolds numbers 


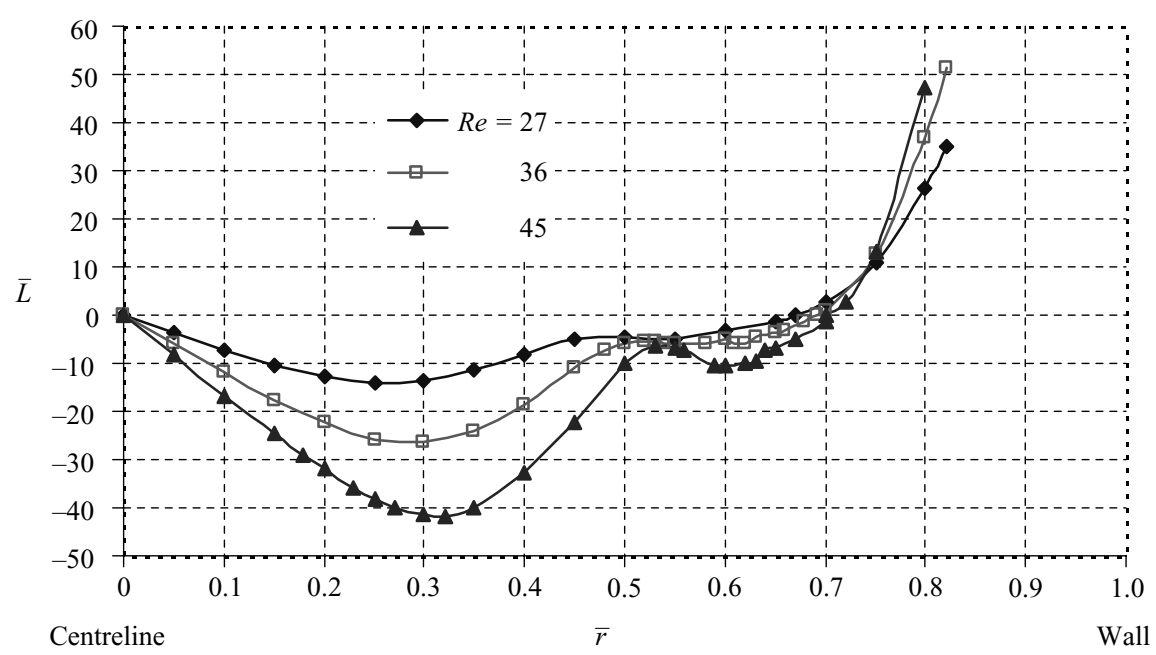

Figure 6 . The dimensionless lift force $\bar{L}$ at different radial positions for a sphere with $\bar{a}=0.15$. The three curves corresponds to $R e=27,36$ and 45, respectively. On each of the curve, there exist two branches on which the slope of the curve is negative.

to converge to a steady state; a refined mesh was necessary to obtain converged results in these simulations. For example, when $R e=1$, the typical mesh used in our simulations consists of $9.09 \times 10^{4}$ elements, $1.28 \times 10^{5}$ nodes and $1.68 \times 10^{4}$ vertices; when $R e=45$, the typical mesh consists of $9.86 \times 10^{4}$ elements, $1.39 \times 10^{5}$ nodes and $1.82 \times 10^{4}$ vertices.

Similar complicated distributions of the lift force can be observed in Asmolov's (1999) calculation of the lift force on a small neutrally buoyant sphere in a plane Poiseuille flow. In his figure 8 , the lift is plotted as a function of $d / l$, where $d$ is the distance from the wall to the particle centre and $l$ is the channel width. Five curves for five channel Reynolds numbers $R_{c}$ (defined in $\left.\S 1\right)=15,100,300,1000$ and 3000 are plotted. There is only one negative-slope branch on the curves for $R_{c}=15$ and 100, which is at the centreline. There are two negative-slope branches on the curves for $R_{c}=300,1000$ and 3000. One of the branches is at the centreline and the other is between the centreline and the wall. Both our numerical simulation and Asmolov's (1999) calculation using matched asymptotic methods show that at high $R e$, there exists a local minimum of the lift force between the centreline and the stable equilibrium position. This observation prompts us to consider the possibility of multiple equilibrium positions for neutrally buoyant particles in Poiseuille flows.

Matas, Morris \& Guazzelli (2004) measured lateral migration of dilute suspensions of neutrally buoyant spheres in pipe flows; they observed single equilibrium positions when $\bar{U}_{m}$ is low $(60,350)$ and multiple equilibrium positions when $\bar{U}_{m}$ is high $(760)$. In their experiments using spheres with $\bar{a}=0.06875$ in flows with $\bar{U}_{m}=760$, they observed a first equilibrium position close to the wall and a second equilibrium position at $\bar{r}=0.5 \pm 0.2$. They also reported that when $\bar{U}_{m}$ is increased to 1500 , the first equilibrium position close to the wall disappeared and the second equilibrium position became the only equilibrium position. Matas et al. proposed to explain the second equilibrium position using the change of convexity in the lift force profiles obtained from the matched asymptotic methods. They argued that the particles could accumulate in the region near the local minimum of the lift force. 


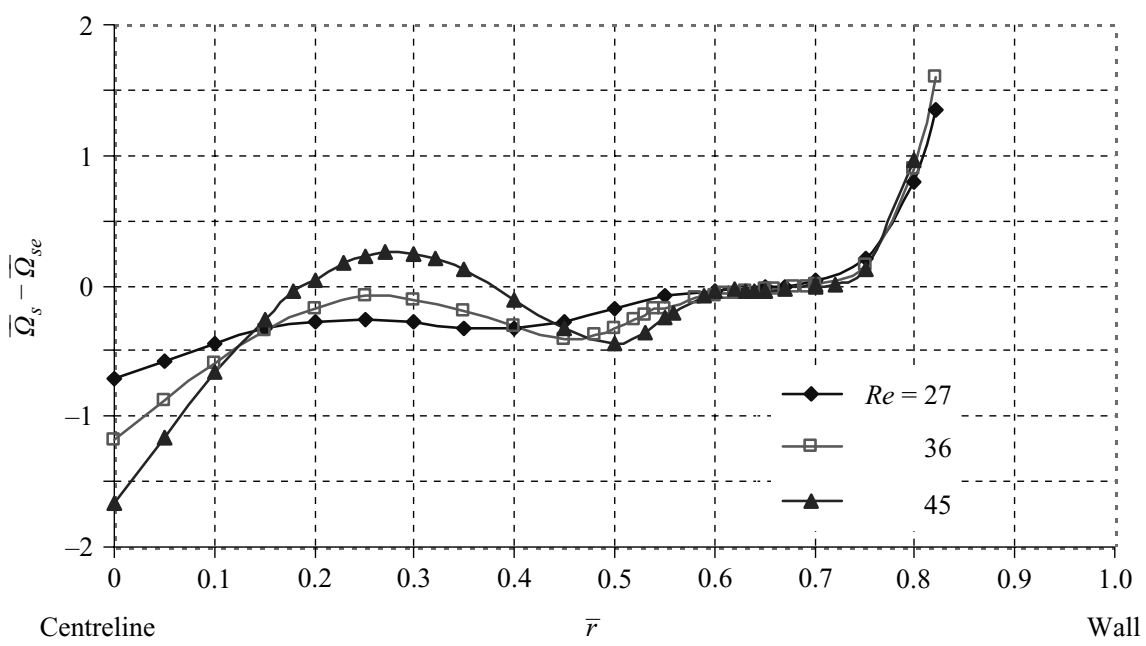

Figure 7. The dimensionless slip angular velocity discrepancy at different radial positions for a sphere with $\bar{a}=0.15$. The three curves correspond to $R e=27,36$ and 45 , respectively.

In numerical simulations, Patankar et al. (2001a) reported multiple equilibrium positions for particles heavier than the fluid in plane Poiseuille flows perpendicular to the gravity. We have not obtained multiple equilibrium positions in simulations for a neutrally buoyant sphere in pipe flows. We are not able to run simulations matching the experimental conditions under which multiple equilibrium positions were observed. From figure 6, it seems possible that a second stable equilibrium position appears near $\bar{r}=0.55$ at higher $R e$. It is also possible that the second equilibrium position arises from particle interaction in the experiments. The existence of multiple equilibrium positions for a single neutrally buoyant particle in a pipe flow is still an open question.

The $\bar{\Omega}_{s}-\bar{\Omega}_{s e}$ vs. $\bar{r}$ curves for the cases with $\bar{a}=0.15$ and $R e=27,36$ or 45 are plotted in figure 7. Our assertion that the lift force changes sign with the discrepancy $\bar{\Omega}_{s}-\bar{\Omega}_{s e}$ remains true for $R e=27$ and 36 but not for $R e=45$. When $R e=45, \bar{\Omega}_{s}-\bar{\Omega}_{s e}$ changes sign twice in the range $0<\bar{r}<0.5$ (figure 7); however, the lift force remains negative in the same range (figure 6). Our assertion is true in the vicinity of the stable equilibrium position at all the Reynolds numbers, which will be the region in which the lift correlation is developed.

We seek correlations between the lift force $\bar{L}$ and the product

$$
F=\bar{U}_{s}\left(\bar{\Omega}_{s}-\bar{\Omega}_{s e}\right) .
$$

From our data, we noted that in the vicinity of the stable equilibrium position, the relation between $\bar{L}$ and $F$ may be represented by a linear correlation:

$$
\bar{L}(\bar{r}, \operatorname{Re}, \bar{a})=k(\operatorname{Re}, \bar{a}) F(\bar{r}, \operatorname{Re}, \bar{a}),
$$

where $k$ is the proportionality coefficient which depends on the Reynolds number and the radius ratio $\bar{a}$. Some examples of the linear correlation between $\bar{L}$ and $F$ are plotted in figure 8 and the values of $k$ are given in table 2 . The linear correlation (4.2) is not valid far away from the equilibrium position; we also list the range of the radial position in which the linear correlation is valid in table 2.

The value of $k$ decreases as $R e$ increases when the Reynolds number is low. However, $k$ increases as $R e$ increases when $R e \geqslant 27$. This change is possibly related 
(a)

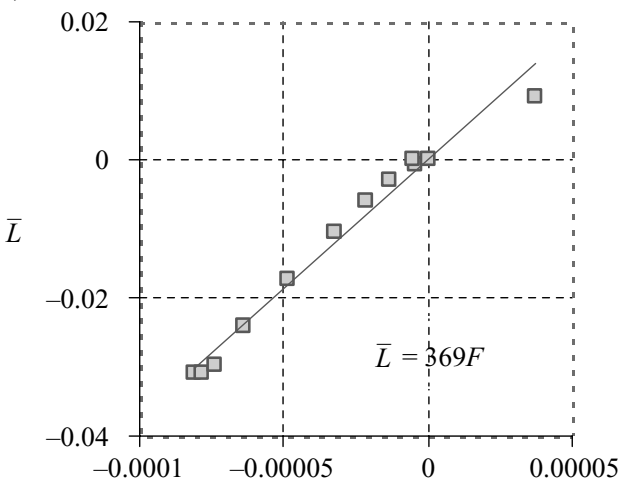

(c)

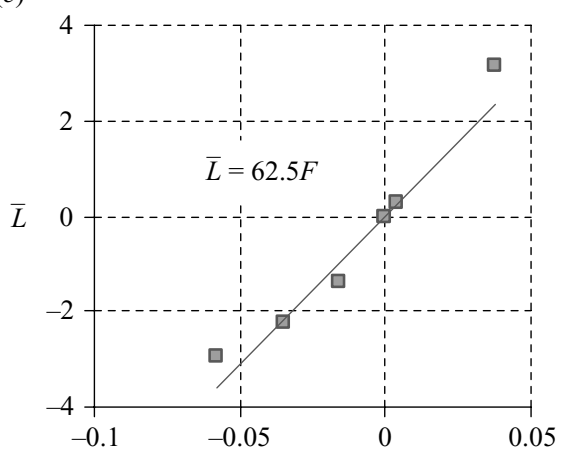

(e)

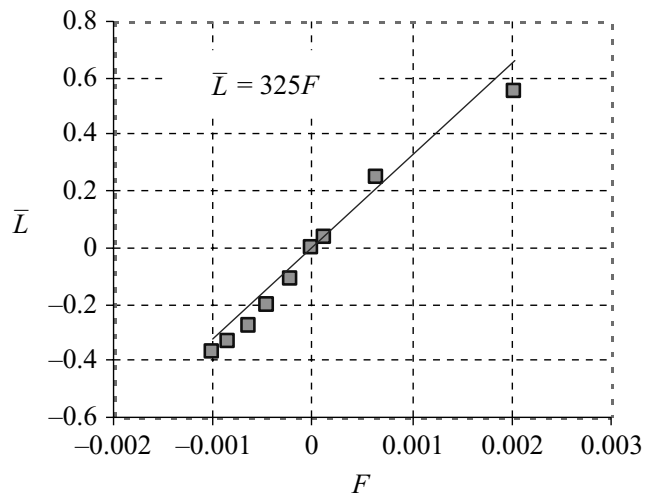

(b)

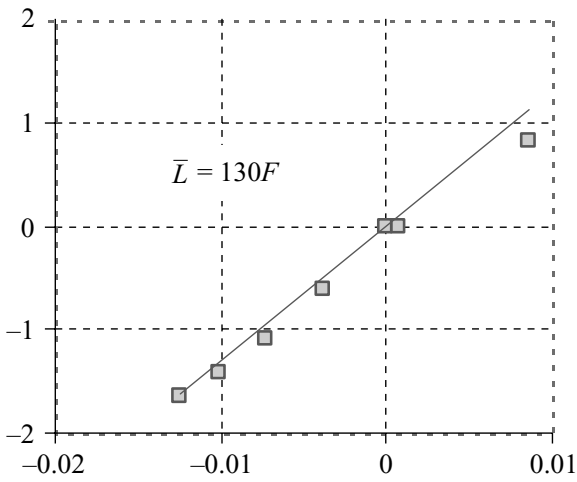

(d)

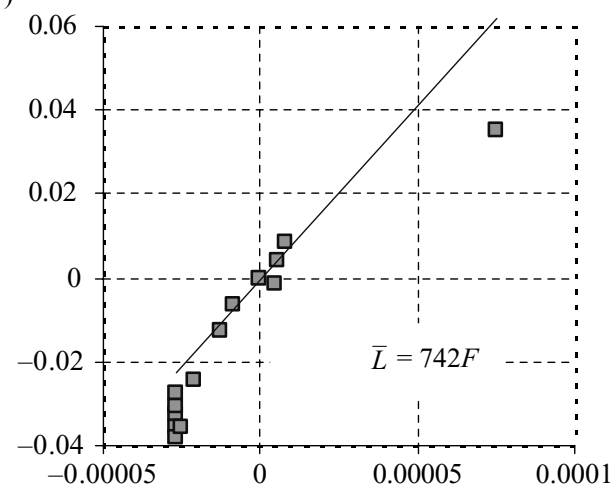

(f)

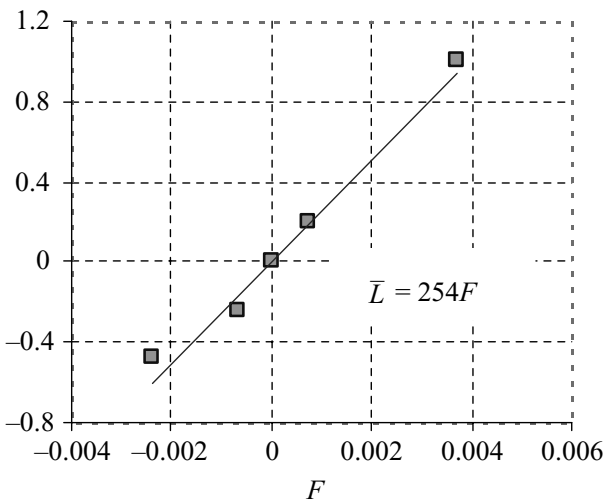

FIGURE 8 . The linear correlation between $\bar{L}$ and $F$ in the vicinity of the stable equilibrium position of a neutrally buoyant particle. (a) $R e=1, \bar{a}=0.15$; (b) $\operatorname{Re}=9, \bar{a}=0.15$; (c) $\operatorname{Re}=18$, $\bar{a}=0.15 ;(d) \operatorname{Re}=1, \bar{a}=0.1 ;(e) \operatorname{Re}=4, \bar{a}=0.1 ;(f) \operatorname{Re}=8, \bar{a}=0.1$.

to the emergence of the second negative-slope branch on the $\bar{L}$ vs. $\bar{r}$ plot at high Reynolds numbers. We shall focus on the low-Reynolds-number cases and data-fitting analyses lead to expressions for $k$ in terms of the Reynolds number:

$$
\begin{aligned}
& k=809 R e^{-0.604} \text { for } \bar{a}=0.1,1 \leqslant R e \leqslant 12, \\
& k=450 R e^{-0.658} \text { for } \bar{a}=0.15,1 \leqslant R e \leqslant 22.5 .
\end{aligned}
$$




\begin{tabular}{|c|c|c|c|c|}
\hline $\bar{a}$ & $R e$ & $k$ & Equilibrium position $\bar{r}_{e}$ & Range of the linear correlation \\
\hline \multirow[t]{8}{*}{0.1} & 1 & 742 & 0.603 & $0.2 \leqslant \bar{r} \leqslant 0.7$ \\
\hline & 2 & 620 & 0.608 & $0.3 \leqslant \bar{r} \leqslant 0.7$ \\
\hline & 4 & 325 & 0.638 & $0.4 \leqslant \bar{r} \leqslant 0.75$ \\
\hline & 6 & 268 & 0.661 & $0.55 \leqslant \bar{r} \leqslant 0.75$ \\
\hline & 8 & 254 & 0.674 & $0.6 \leqslant \bar{r} \leqslant 0.75$ \\
\hline & 10 & 201 & 0.684 & $0.6 \leqslant \bar{r} \leqslant 0.75$ \\
\hline & 12 & 169 & 0.708 & $0.65 \leqslant \bar{r} \leqslant 0.8$ \\
\hline & 1 & 369 & 0.573 & $0.3 \leqslant \bar{r} \leqslant 0.6$ \\
\hline \multirow[t]{8}{*}{0.15} & 2 & 346 & 0.573 & $0.3 \leqslant \bar{r} \leqslant 0.6$ \\
\hline & 9 & 130 & 0.601 & $0.4 \leqslant \bar{r} \leqslant 0.65$ \\
\hline & 13.5 & 77.2 & 0.623 & $0.45 \leqslant \bar{r} \leqslant 0.7$ \\
\hline & 18 & 62.5 & 0.642 & $0.5 \leqslant \bar{r} \leqslant 0.7$ \\
\hline & 22.5 & 53.5 & 0.657 & $0.55 \leqslant \bar{r} \leqslant 0.7$ \\
\hline & 27 & 56.5 & 0.670 & $0.6 \leqslant \bar{r} \leqslant 0.75$ \\
\hline & 36 & 69.8 & 0.691 & $0.65 \leqslant \bar{r} \leqslant 0.75$ \\
\hline & 45 & 85.2 & 0.700 & $0.67 \leqslant \bar{r} \leqslant 0.75$ \\
\hline
\end{tabular}

TABLE 2. The values of the proportionality coefficient $k$ and the equilibrium position $\overline{\boldsymbol{r}}_{e}$ as functions of the radius ratio $\bar{a}$ and $R e$. The range of the radial position in which the linear correlation (4.2) is valid is also listed.

Inserting the expression of $k$ into the linear correlation (4.2), we can obtain the correlations between $\bar{L}$ and $F$. To reveal the dependence of the lift force on the slip velocities explicitly, we substitute the definitions of $\bar{L}$ and $F$ into the linear correlation and it follows that

$$
\begin{aligned}
& L=424 R e^{-0.604} \rho_{f} U_{s}\left(\Omega_{s}-\Omega_{s e}\right)(2 a)^{3} \text { for } \bar{a}=0.1, \\
& L=236 \operatorname{Re}^{-0.658} \rho_{f} U_{s}\left(\Omega_{s}-\Omega_{s e}\right)(2 a)^{3} \text { for } \bar{a}=0.15 .
\end{aligned}
$$

Both of these correlations are analogous to the lift correlation we obtained in the two-dimensional cases (Wang \& Joseph 2003):

$$
L=182.6 R e^{-0.515} \rho_{f} U_{s}\left(\Omega_{s}-\Omega_{s e}\right)(2 a)^{2},
$$

which is for the lift force per unit length on a cylindrical particle whose diameter is $1 / 12$ of the channel width. It is noted that the exponent of the Reynolds number is -0.604 in (4.5) and it is closer to the value -0.515 in (4.7).

The lift force in our correlation is on a freely rotating particle translating at steady velocity. Thus, correlations (4.5) and (4.6) apply to particles with zero acceleration. For a migrating particle with substantial acceleration, correlations (4.5) and (4.6) may not be valid.

4.2. Correlations for the slip velocity $U_{s}$ and slip angular velocity $\Omega_{s}$

Besides the lift force on the particle, the translational and angular velocities of the particle at steady state are also of interest. We shall construct correlations for the slip velocity $U_{s}$ and slip angular velocity $\Omega_{s}$ from constrained simulations; the particle velocities can then be easily computed using (1.1) and (1.2). The correlations for $U_{s}$ and $\Omega_{s}$ are necessary to compute the lift force, because they appear in the lift correlations (4.5) and (4.6).

We illustrate the correlation construction for a particle with the radius ratio $\bar{a}=0.15$. We plot the Reynolds number $\bar{\Omega}_{s}$ on $\log$-log plots versus $R e$; straight lines for each value of $\bar{r}$ are obtained. Similar straight lines on $\log -\log$ plots are obtained when we 
(a)

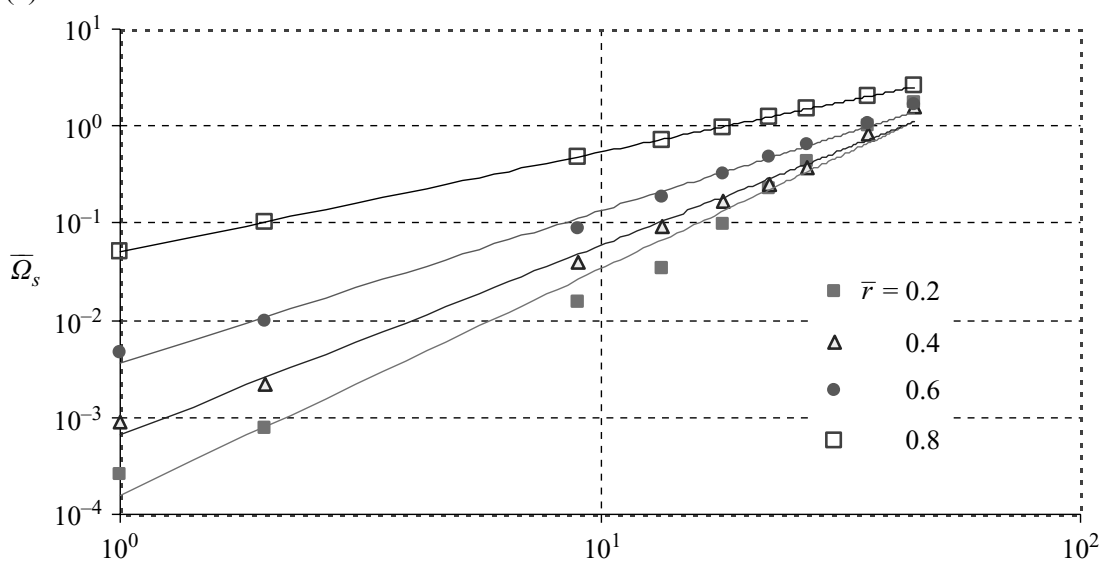

(b)

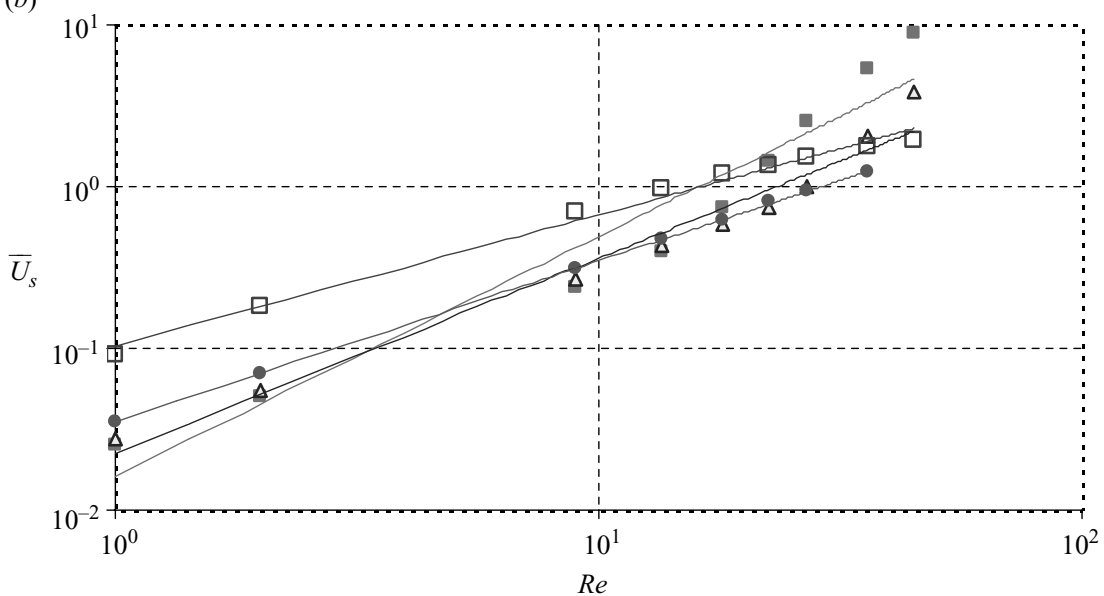

FIGURE 9. Power law correlations of $(a) \bar{\Omega}_{s} v$ s. Re and $(b) \bar{U}_{s} v$ s. Re at different values of $\bar{r}$ for a sphere with $\bar{a}=0.15$.

plot $\bar{U}_{s}$ versus $R e$. Examples of such plots are shown in figures $9(a)$ and $9(b)$ for $\bar{\Omega}_{s}$ vs. $R e$ and $\bar{U}_{s} v s$. $R e$, respectively.

Power law correlations arise from the straight lines in $\log -\log$ plots for $\bar{\Omega}_{s}$ and $\bar{U}_{s}$

$$
\begin{aligned}
& \bar{U}_{s}(\bar{r}, \operatorname{Re}, \bar{a})=b(\bar{r}, \bar{a}) \operatorname{Re} e^{m(\bar{r}, \bar{a})}, \\
& \bar{\Omega}_{s}(\bar{r}, \operatorname{Re}, \bar{a})=c(\bar{r}, \bar{a}) \operatorname{Re}^{n(\bar{r}, \bar{a})}
\end{aligned}
$$

The coefficients $b, m, c$ and $n$ are obtained for a particle with $\bar{a}=0.15$ in the range $0.1 \leqslant \bar{r} \leqslant 0.8$ and plotted in figure 10. Exponential fitting and linear fitting may give reasonable approximations to the prefactors $b$ and $c$ and exponents $m$ and $n$, respectively:

$$
\begin{array}{cl}
b=1.1 \times 10^{-2} \exp (2.2 \bar{r}), & c=2.1 \times 10^{-5} \exp (9.2 \bar{r}), \\
m=-1.1 \bar{r}+1.7, \quad n=-2.1 \bar{r}+2.8 .
\end{array}
$$




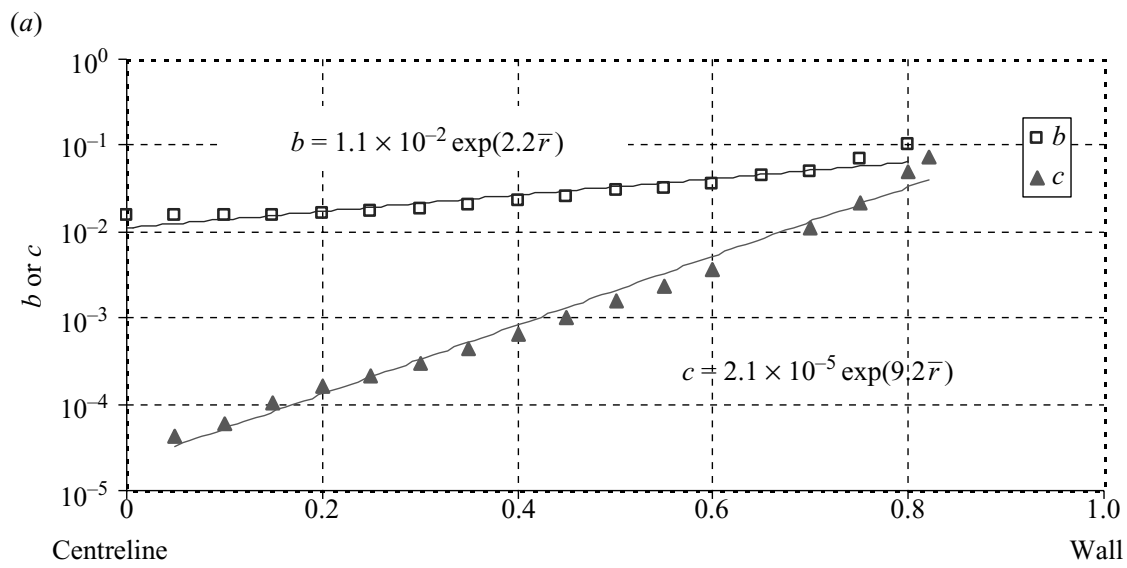

(b)

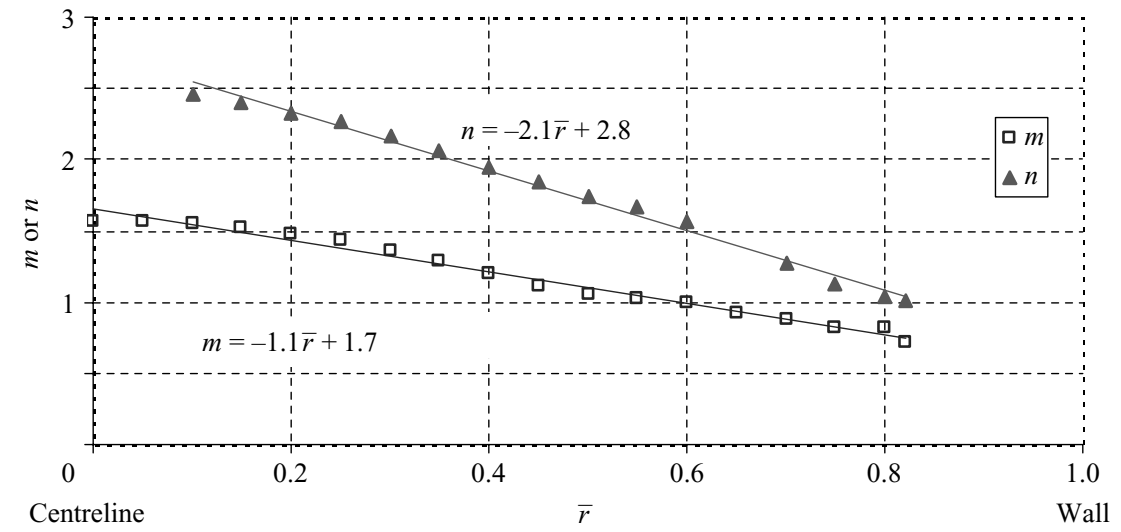

FIgURE 10. The prefactors $b$ and $c$ and exponents $m$ and $n$ in correlations (4.8) and (4.9) for a particle with $\bar{a}=0.15$ in the range $0.1 \leqslant \bar{r} \leqslant 0.8$.

Substitution of (4.10) and (4.11) into (4.8) and (4.9) leads to explicit expressions for $\bar{U}_{s}$ and $\bar{\Omega}_{s}$

$$
\begin{aligned}
& \bar{U}_{s}=1.1 \times 10^{-2} \exp (2.2 \bar{r}) R e^{-1.1 \bar{r}+1.7}, \\
& \bar{\Omega}_{s}=2.1 \times 10^{-5} \exp (9.2 \bar{r}) R e^{-2.1 \bar{r}+2.8},
\end{aligned}
$$

which apply to a particle with $\bar{a}=0.15$ in the range $0.1 \leqslant \bar{r} \leqslant 0.8$. Correlation (4.12) and (4.13) are generally valid in the range of $1 \leqslant R e \leqslant 45$. However, correlation (4.12) is not in good agreement with the data for $\bar{U}_{s}$ on the second negative-slope branch on the $\bar{L} v$ s. $\bar{r}$ curve $(0.5 \leqslant \bar{r} \leqslant 0.65)$ at high Reynolds numbers $(R e=27,36$ and 45$)$. Thus, correlation (4.12) in the range $0.5 \leqslant \bar{r} \leqslant 0.65$ is valid only for $1 \leqslant R e \leqslant 22.5$.

Correlations for $\bar{U}_{s}$ and $\bar{\Omega}_{s}$ for $\bar{a}=0.1$ are developed using the same procedure as for $\bar{a}=0.15$. We omit the details of derivation and give only the final formulae for $\bar{U}_{s}$ and $\bar{\Omega}_{s}$

$$
\begin{aligned}
& \bar{U}_{s}=8.3 \times 10^{-3} \exp (1.8 \bar{r}) R e^{-1.4 \bar{r}+1.9}, \\
& \bar{\Omega}_{s}=7.7 \times 10^{-6} \exp (9.0 \bar{r}) R e^{-3.3 \bar{r}+3.8},
\end{aligned}
$$

which apply to a particle with $\bar{a}=0.1$ in the range $0.05 \leqslant \bar{r} \leqslant 0.85$. The range of the Reynolds number in which (4.14) and (4.15) is valid is $1 \leqslant R e \leqslant 12$. 


\begin{tabular}{cccccc}
\hline $\bar{a}$ & $R e$ & $\bar{U}_{m}$ & $\bar{r}_{e}$ & $\bar{\Omega}_{s e}$ & $\bar{U}_{s e}$ \\
0.05 & 2 & 100 & 0.731 & 0.00710 & 0.0247 \\
& 1 & 12.5 & 0.603 & 0.00188 & 0.0219 \\
& 2 & 25 & 0.608 & 0.00509 & 0.0444 \\
0.1 & 4 & 50 & 0.638 & 0.0209 & 0.0901 \\
& 6 & 75 & 0.661 & 0.0498 & 0.152 \\
& 8 & 100 & 0.674 & 0.0901 & 0.470 \\
& 10 & 125 & 0.684 & 0.139 & 0.712 \\
& 12 & 150 & 0.708 & 0.202 & 0.296 \\
& 1 & 5.56 & 0.573 & 0.00354 & 0.0338 \\
& 2 & 11.1 & 0.573 & 0.00765 & 0.0675 \\
0.15 & 13 & 50 & 0.601 & 0.0861 & 0.306 \\
& 18 & 75 & 0.623 & 0.197 & 0.482 \\
& 22.5 & 100 & 0.642 & 0.342 & 0.730 \\
& 27 & 125 & 0.657 & 0.513 & 0.785 \\
0.2 & 36 & 150 & 0.670 & 0.705 & 1.07 \\
0.25 & 45 & 200 & 0.691 & 1.16 & 1.18 \\
& 32 & 250 & 0.700 & 1.67 & 1.74 \\
& 50 & 100 & 0.598 & 0.793 & 2.84
\end{tabular}

TABLE 3. Particle parameters at stable equilibrium: the equilibrium position $\bar{r}_{e}$, the dimensionless slip angular velocity $\bar{\Omega}_{s e}=\rho_{f} \Omega_{s e}(2 a)^{2} / \mu$ and the dimensionless slip velocity $\bar{U}_{s e}=$ $\rho_{f} U_{s e}(2 a) / \mu$.

\subsection{Correlations for the particle parameters at equilibrium}

The equilibrium state of a particle is always the focus of the study of particle migration. We obtain the particle parameters at stable equilibrium, such as the equilibrium position $\bar{r}_{e}$, the slip velocity $U_{s e}$ and the slip angular velocity $\Omega_{s e}$ by unconstrained simulation and find that they may be correlated to the Reynolds number. We summarize the particle parameters at stable equilibrium in table 3.

We can correlate the stable equilibrium position of a neutrally buoyant sphere with the Reynolds number (see figure 11):

$$
\begin{array}{ll}
\bar{r}_{e}=0.591 R e^{0.0644} & \text { for } \bar{a}=0.1, \\
\bar{r}_{e}=0.555 R e^{0.0546} & \text { for } \bar{a}=0.15 .
\end{array}
$$

The equilibrium position $\bar{r}_{e}$ moves closer to the wall as the Reynolds number increases. We also observe that when the flow Reynolds number $\bar{U}_{m}$ is fixed, the larger particle finds its equilibrium position closer to the centreline than the smaller particle. The above observations are in agreement with the experiments by Karnis et al. (1966), who reported that neutrally buoyant particles stabilized closer to the wall for larger flow rates and closer to the centre for larger particles.

Matas et al. (2004) reported that for neutrally buoyant spheres with a diameter $d=900 \mu \mathrm{m}$ in the pipe of diameter $D=8 \mathrm{~mm}$, the equilibrium position is $\bar{r}_{e}=0.64 \pm$ 0.04 for $\bar{U}_{m}=60$ and $\bar{r}_{e}=0.78 \pm 0.04$ for $\bar{U}_{m}=350$. The value of $\bar{a}$ is close to 0.1 in these experiments, thus we can compare correlation (4.16) to the experimental results. Equations (4.16) and (2.15) predict $\bar{r}_{e}=0.654,0.732$ for $\bar{U}_{m}=60,350$, respectively, in good agreement with the experimental results. Matas et al. (2004) stated that the matched asymptotic calculation (Schonberg \& Hinch 1989; Asmolov 1999) predicts that $\bar{r}_{e}=0.71,0.85$ for $\bar{U}_{m}=60,350$, respectively, in both cases larger 


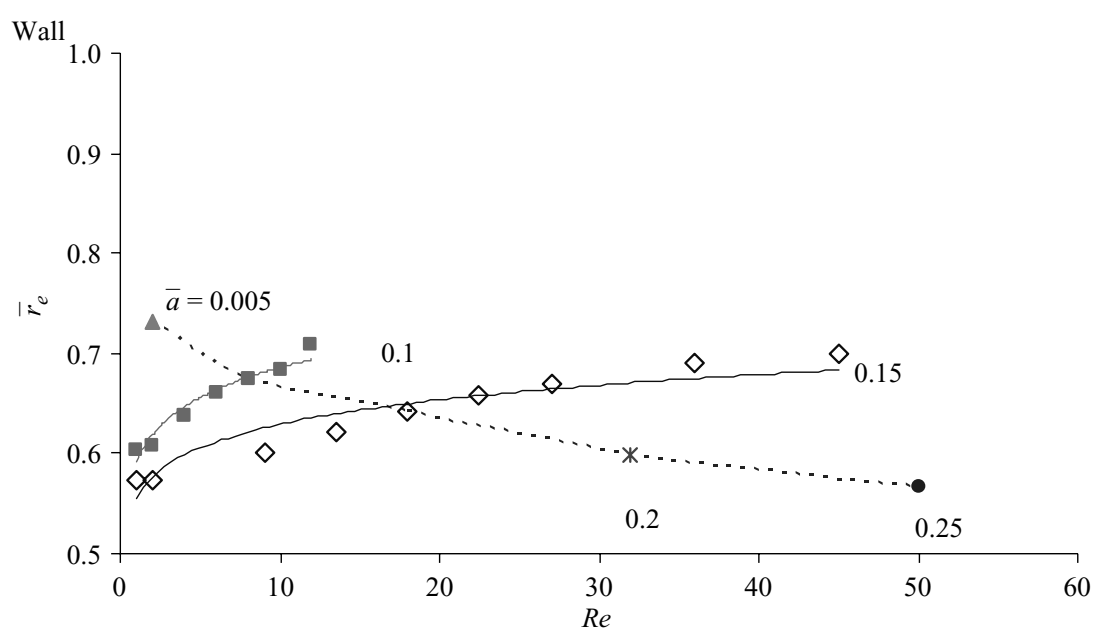

FIGURE 11. The stable equilibrium position $\bar{r}_{e}$ of a neutrally buoyant sphere as a function of the Reynolds number. The two power law curves are for spheres with $\bar{a}=0.1$ and $\bar{a}=0.15$, respectively. $\diamond, \bar{r}_{e}=0.555 R e^{0.0546} ; \mathbf{\square}, \bar{r}_{e}=0.591 R e^{0.0644}$. All the points on the dotted line are for the same flow Reynolds number $\bar{U}_{m}=100$; the dotted line shows that when $\bar{U}_{m}$ is fixed, the equilibrium position becomes closer to the centreline as $\bar{a}$ increases.

than the experimental value. They attributed this difference to the relatively large size of the particles. They reported that when smaller particles $(200 \mu \mathrm{m})$ were used in the experiments, equilibrium positions were closer to the theoretical predictions. The actual values of $\bar{r}_{e}$ for $200 \mu \mathrm{m}$ particles are not reported in their paper, but we can infer that $\bar{r}_{e}$ for $200 \mu \mathrm{m}$ particles is larger than $\bar{r}_{e}$ for $900 \mu \mathrm{m}$ particles at the same $\bar{U}_{m}$. This agrees with our conclusion that the larger particle finds its equilibrium position closer to the centreline than the smaller particle at a fixed $\bar{U}_{m}$.

If we correlate the dimensionless slip angular velocity at equilibrium $\bar{\Omega}_{s e}$ with the Reynolds number $R e$, we obtain (see figure 12)

$$
\bar{\Omega}_{s e}=0.0023 R e^{1.72}, \quad \text { i.e. } \Omega_{s e}=0.0023 R e^{1.72} \mu /\left(\rho_{f} 4 a^{2}\right) .
$$

This correlation appears to be applicable to a wide range of the radius ratios: $0.05 \leqslant \bar{a} \leqslant 0.25$. Correlation (4.18) is important because it gives explicitly the slip angular velocity when the particle is at stable equilibrium.

Now with all the correlations for $k, \bar{U}_{s}, \bar{\Omega}_{s}$ and $\bar{\Omega}_{s e}$ available, we are in a position to make the lift correlation (4.2) completely explicit. Take a particle with $\bar{a}=0.15$, for example,

$$
\bar{L}=k \bar{U}_{s}\left(\bar{\Omega}_{s}-\bar{\Omega}_{s e}\right),
$$

where $k, \bar{U}_{s}, \bar{\Omega}_{s}$ and $\bar{\Omega}_{s e}$ are given in (4.4), (4.12), (4.13) and (4.18), respectively. Therefore, we are able to compute the lift force on a particle at different radial positions from the Reynolds number and radius ratio. It should be noted that the lift force applies to a freely rotating particle translating at a steady speed.

If we set $\bar{L}$ in (4.19) to zero, we can solve for the equilibrium position $\bar{r}_{e}$. The value $\bar{L}=0$ is given by $\bar{\Omega}_{s}=\bar{\Omega}_{s e}$; this leads to a formula for the equilibrium position

$$
\bar{r}_{e}=\frac{4.6-0.98 \ln R e}{8.8-2.1 \ln R e}
$$




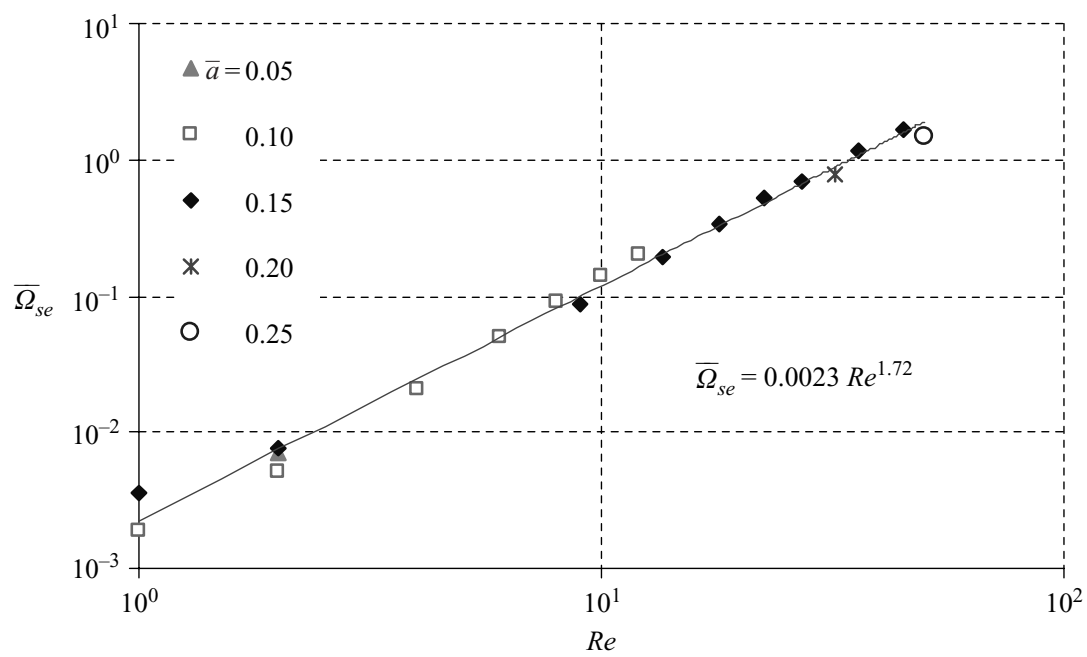

FIGURE 12. The correlation between $\bar{\Omega}_{s e}$ and the Reynolds number $R e$.

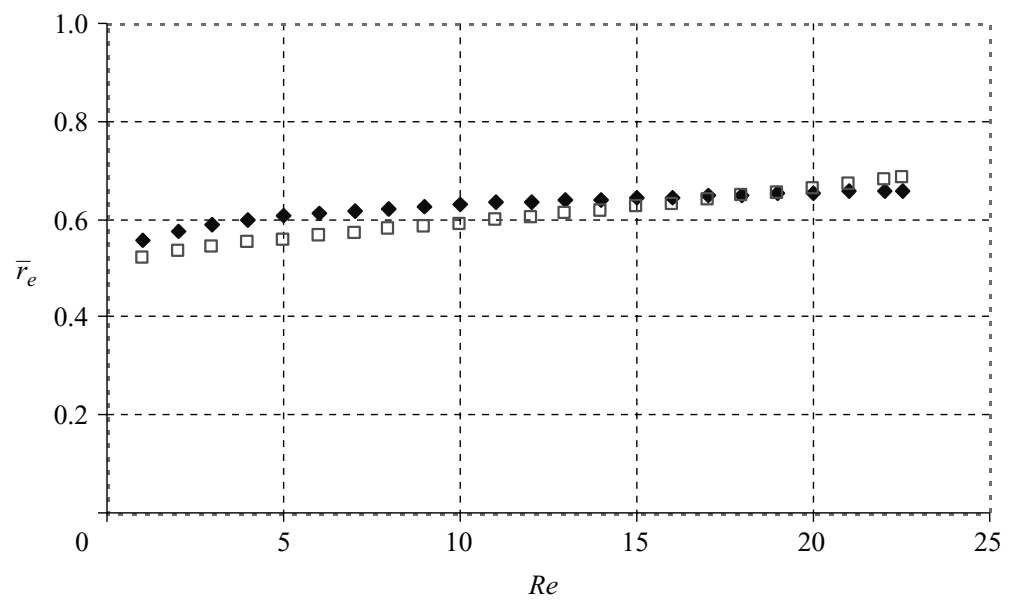

Figure 13. The values of $\bar{r}_{e}$ computed by $\bullet,(4.17)$ and $\square,(4.20)$ in the range $1 \leqslant R e \leqslant 22.5$ for $\bar{a}=0.15$.

for a particle with $\bar{a}=0.15$ which can be compared to correlation (4.17). Equations (4.17) and (4.20) give rise to similar values for $\bar{r}_{e}$ in the range $1 \leqslant R e \leqslant 22.5$ (see figure 13); (4.17) gives a better approximation to the simulation results because it is directly developed for $\bar{r}_{e}$. When $R e>22.5$, the agreement is not good between (4.17) and (4.20).

A summary of our correlations is presented in table 4. Correlation of formulae exhibiting the dependence of prefactors and exponents on $\bar{a}$ requires more computation.

\section{Comparison of lift expressions}

Wang \& Joseph (2003) compared the lift correlation (4.7) with analytical lift expressions in the literature. Their comparison was limited because the correlation (4.7) is for a two-dimensional cylindrical particle, whereas the lift expressions of 
$\bar{L}(\bar{r} ; \bar{a}, R e)=k(\bar{a}, \operatorname{Re}) \bar{U}_{s}\left(\bar{\Omega}_{s}-\bar{\Omega}_{s e}\right)$

where $k(\bar{a}, R e)=g(\bar{a}) R e^{-h(\bar{a})}$

$\bar{U}_{s}=b(\bar{r} ; \bar{a}) R e^{m(\bar{r} ; \bar{a})}$

$\bar{U}_{s}=1.1 \times 10^{-2} \exp (2.2 \bar{r}) R e^{-1.1 \bar{r}+1.7}$ for $\bar{a}=0.15$

$\bar{U}_{s}=8.3 \times 10^{-3} \exp (1.8 \bar{r}) R e^{-1.4 \bar{r}+1.9}$ for $\bar{a}=0.1$

$\bar{\Omega}_{s}=c(\bar{r} ; \bar{a}) R e^{n(\bar{r} ; \bar{a})}$

$\bar{\Omega}_{s}=2.1 \times 10^{-5} \exp (9.2 \bar{r}) R e^{-2.1 \bar{r}+2.8}$ for $\bar{a}=0.15$

$\bar{\Omega}_{s}=7.7 \times 10^{-6} \exp (9.0 \bar{r}) R e^{-3.3 \bar{r}+3.8}$ for $\bar{a}=0.1$

$\bar{\Omega}_{s e}=0.0023 R e^{1.72}$

$\bar{r}_{e}=f(\bar{a}) R e^{q(\bar{a})}$

\begin{tabular}{|c|c|c|}
\hline \multicolumn{3}{|c|}{ Lift law } \\
\hline $\bar{a}$ & $g(\bar{a})$ & $h(\bar{a})$ \\
\hline 0.1 & 809 & 0.604 \\
\hline 0.15 & 450 & 0.65 \\
\hline
\end{tabular}

$0.05 \leqslant \bar{a} \leqslant 0.25$

applicable to a wide range of $\bar{a}$

$\begin{array}{ccc}\bar{a} & f(\bar{a}) & q(\bar{a}) \\ 0.1 & 0.591 & 0.0644 \\ 0.15 & 0.555 & 0.0546\end{array}$

TABLE 4. Structure of the correlations for the lift law.

Saffman (1965, 1968) and Auton (1987) are for a sphere. A comparison between correlations (4.5) and (4.6) with the aforementioned analytical lift expressions is more sensible; though the comparison is still tentative because the analytical lift expressions are for a particle in a linear shear flow on an unbounded domain whereas our threedimensional simulation is in a tube Poiseuille flow. We will compare the correlation (4.5) to the analytical lift expressions with these caveats in mind.

Auton (1987) derived a lift expression for a fixed sphere in an inviscid fluid in which uniform motion is perturbed by a weak shear:

$$
\boldsymbol{L}=\frac{2}{3} \pi a^{3} \rho \boldsymbol{\omega} \times(\boldsymbol{u}-\boldsymbol{U}) .
$$

In the case under consideration, $\boldsymbol{\omega}=\boldsymbol{e}_{z} \dot{\gamma}$ and $\boldsymbol{u}-\boldsymbol{U}=\boldsymbol{e}_{\boldsymbol{x}} U_{s}$; the lift force in the $y$-direction is

$$
L=\frac{2}{3} \pi a^{3} \rho U_{s} \dot{\gamma},
$$

which is similar to our correlation (4.5), but differs from it in several ways: (5.2) has a constant prefactor for inviscid fluids, whereas viscous effects enter into (4.5) through $R e$; the lift force depends on $\dot{\gamma}$ in (5.2), but on the discrepancy $\Omega_{s}-\Omega_{\text {se }}$ in (4.5); (5.2) is for a fixed three-dimensional sphere while (4.5) is for a freely rotating sphere with zero acceleration.

Saffman $(1965,1968)$ obtained an expression for the lift force on a rotating sphere in an unbounded linear shear flow at indefinitely small Reynolds number:

$$
L=6.46 \rho_{f}^{0.5} \mu^{0.5} U_{s} \dot{\gamma}^{0.5} a^{2}+\text { lower-order terms. }
$$

If we make following changes in (4.5): $\operatorname{Re}=\rho_{f} \dot{\gamma}_{w}(2 a)^{2} / \mu$, the power of $\operatorname{Re}(-0.604) \rightarrow$ $(-0.5)$, then (4.5) becomes

$$
L=1696 \rho_{f}^{0.5} \mu^{0.5} U_{s} \dot{\gamma}_{w}^{-0.5}\left(\Omega_{s}-\Omega_{s e}\right) a^{2} .
$$

Comparing (5.4) and the leading term in (5.3), we note that both expressions are linear in $U_{s}$; both have a similar dependence on $\rho_{f}, \mu$ and $a$. However, the dependence on $\dot{\gamma}$ and $\Omega_{s}-\Omega_{s e}$ is greatly different. 
For a neutrally buoyant particle at equilibrium, $L=0$ and (5.2) and (5.3) imply $U_{s}=0$. The Auton and Saffman formulae thus predict that the slip velocity is zero for a neutrally buoyant sphere at equilibrium.

McLaughlin (1991) generalized Saffman's analysis to remove the restriction that the Reynolds number $\bar{U}_{s}$ based on slip velocity $U_{s}$ is much smaller than the square root of the Reynolds number Re based on the shear rate and derived an expression for the lift force:

where

$$
L=\frac{6.46}{2.255} \rho_{f}^{0.5} \mu^{0.5} U_{s} \dot{\gamma}^{0.5} a^{2} J(\varepsilon),
$$

$$
\varepsilon=\frac{\sqrt{R e}}{\bar{U}_{s}}, \quad R e=\frac{\rho_{f} \dot{\gamma}(2 a)^{2}}{\mu}, \quad \bar{U}_{s}=\frac{\rho_{f} U_{s}(2 a)}{\mu},
$$

and $J$ is a function of $\varepsilon$ only and has a value of 2.255 as $\varepsilon \rightarrow \infty$ (the Saffman limit). Equation (5.5) shows that zero lift force is obtained when $U_{s}=0$ or $J(\varepsilon)=0$. The solution provided by McLaughlin gives $J(\varepsilon)=0$ at $\varepsilon=0.215$, i.e. $\bar{U}_{s}=\sqrt{\operatorname{Re}} / 0.215$. Hence, $U_{s}$ is not single valued for $L=0$.

In the lift expressions given by Auton, Saffman and McLaughlin, zero lift force is determined by the slip velocity $U_{s}$. This is not the case in our simulation for a sphere in tube Poiseuille flows; our results show that $\Omega_{s}=\Omega_{s e}$ gives rise to zero lift. The difference may be because linear shear flow has a zero shear gradient whereas the shear gradient in Poiseuille flow is a constant and not small.

\section{Conclusion and discussion}

(i) The motion of a single neutrally buoyant rigid sphere in tube Poiseuille flow is simulated by two methods: an ALE arbitrary Lagrangian-Eulerian scheme with a moving adaptive mesh; and a DLM distributed Lagrange multiplier/fictitious domain method. The two methods give the same results, and the simulation agrees with experiments.

(ii) A lift law $L=C U_{s}\left(\Omega_{s}-\Omega_{s e}\right)$ analogous to $L=\rho U \Gamma$ of classical aerodynamics which was proposed and validated in two dimensions is validated in three dimensions here; $U_{s}$ is the slip velocity and it is positive, $\Omega_{s}$ is the slip angular velocity and $\Omega_{s e}$ is the slip angular velocity when the sphere is in equilibrium at the Segré-Silberberg radius.

(iii) The slip angular velocity discrepancy $\Omega_{s}-\Omega_{s e}$ is the circulation for the free particle and it is shown to change sign with the lift.

(iv) Numerical experiments using the method of constrained simulation generate data for the lift force and velocities of a freely rotating sphere in steady flows arising from initial-value problems in which the sphere is constrained to move at a fixed radius.

(v) Constrained simulations are very efficient. The lift and all velocities are obtained for different radii at each specified Reynolds number and radius ratio $\bar{a}=a / R$.

(vi) Equilibrium may be identified at the Segré-Silberberg radius at which the lift vanishes (for a neutrally buoyant particle). The equilibrium slip angular velocity is the slip angular velocity at this equilibrium radius.

(vii) Data generated by constrained simulations are processed for straight lines in $\log -\log$ plots and give rise to explicit power-law formulae for all the quantities in the lift law as a function of $R e$ and $\bar{a}$. We go from data to formulae. 
(viii) The equilibrium position (the Segre-Silberberg radius) moves towards the wall as $R e$ increases at each fixed $\bar{a}$; it moves towards the centreline as $\bar{a}$ increases at a fixed flow Reynolds number $\bar{U}_{m}$.

(ix) Our correlations are compared with analytical lift formulae in the literature. None of the analytic formulae for lift change sign at the Segré-Silberberg radius. These formulae also leave the form of the slip velocity $U_{s}$ obscure.

The lift law we have proposed for free circular and spherical particles is analogous to the aerodynamic lift law $\rho U \Gamma$ in the case of a rotating circle for which $\Gamma=2 \pi a^{2} \Omega$. It is probable that the lift law for free bodies of more general shape is in the form $L=C U_{s} \Gamma_{s}$ where $C$ depends on fluid properties and geometric parameters and $\Gamma_{s}$ is unknown. The determination of $\Gamma$ even in aerodynamic theory is a complicated problem. In airfoil theory, $\Gamma$ is strongly coordinated with the attack angle of the airfoil. A similar coordination of the circulation with the attack angle is apparently generated by the motion of a free ellipse in a plane Poiseuille flow (Feng, Huang \& Joseph 1995). This problem could be framed in terms of the equilibrium position and orientation of an ellipse in a plane Poiseuille flow. As in the case of circular particles, the equilibrium position is decided by a balance of buoyant weight and lift, where the lift arises as a competition of forces arising from shear gradients and wall effects. This problem ought to be studied by the technique of constrained simulation discussed here. At each fixed $y$, the motion of the ellipse would evolve to a steady state with a fixed angle of attack. This lift on the ellipse at this $y$ could be computed and, of course, as in the case of circular and spherical particles, this lift would balance the buoyant weight, zero for neutrally buoyant particles, at equilibrium, with a certain equilibrium attack angle. The lift must change sign with the attack angle discrepancy.

This paper aims at presenting a general procedure and data structure for the interrogation of numerical simulation data. Our goal is to draw explicit formulae from numerical data, which may be used to model complex problems, obviating further expensive computation. The procedure involves identifying controlling dimensionless groups and data-fitting analyses which lead to expressions for the quantities of interest in terms of prescribed parameters. We believe when properly used, this procedure may help to reveal the underlying physics of the problem and generate practically useful formulae at the same time.

This work was partially supported by the National Science Foundation KDI/New Computational Challenge grant (NSF/CTS-98-73236); by the DOE, Department of Basic Energy Sciences; by a grant from the Schlumberger foundation; from STIMLAB Inc.; and by the Minnesota Supercomputer Institute.

\section{REFERENCES}

Asmolov, E. S. 1999 The inertial lift on a spherical particle in a plane Poiseuille flow at large channel Reynolds number. J. Fluid Mech. 381, 63-87.

Auton, T. R. 1987 The lift force on a spherical body in a rotational flow. J. Fluid Mech. 183, 199-218.

Bretherton, F. P. 1962 Slow viscous motion round a cylinder in a simple shear. J. Fluid Mech. 12, 591-613.

Chor, H. G. \& Joseph, D. D. 2001 Fluidization by lift of 300 circular particles in plane Poiseuille flow by direct numerical simulation. J. Fluid Mech. 438, 101-128.

Feng, J., Hu, H. H. \& JoSEPH, D. D. 1994 Direct simulation of initial values problems for the motion of solid bodies in a Newtonian fluid. Part 2. Couette and Poiseuille flows. J. Fluid Mech. 277, 271-301. 
Feng, J., Huang, P. Y. \& JosePh, D. D. 1995 Dynamic simulation of the motion of capsules in pipelines. J. Fluid Mech. 286, 201-227.

Glowinski, R. 2003 Finite Element Methods for Incompressible Viscous Flow. Handbook of Numerical Analysis (ed., P. G. Ciarlet \& J. L. Lions), North-Holland, Amsterdam Vol. 9, chaps. 8, 9, pp. 3-1176).

Glowinski, R., Pan, T.-W., Hesla, T. I. \& JosePh, D. D. 1999a A distributed Lagrange multiplier/ fictitious domain method for particulate flows. Intl J. Multiphase Flow 25, 755-794.

Glowinski, R., Pan, T.-W., Hesla, T. I., Joseph, D. D. \& Periaux, J. $1999 b$ A distributed Lagrange multiplier/fictitious domain method for flow around moving rigid bodies: application to particulate flow. Intl J. Numer. Meth. Fluids 30, 1043-1066.

Glowinski, R., Pan, T.-W., Hesla, T. I., Joseph, D. D. \& Periaux, J. 2001 A fictitious domain approach to the direct numerical simulation of incompressible fluid flow past moving rigid bodies: application to particulate flow. J. Comput. Phys. 162, 363-426.

Glowinski, R., Pan, T.-W. \& Periaux, J. 1998 Distributed Lagrange multiplier methods for incompressible viscous flow around moving rigid bodies. Comput. Math. Applic. Mech. Engng 151, 181-194.

Goldsmith, H. L. \& Mason, S. G. 1967 The microrheology of dispersions. In Rheology: Theory and Applications (ed. F. R. Eirich), vol. 4, pp. 87-205. Academic.

Hu, H. H., Joseph, D. D. \& Crochet, M. J. 1992 Direct simulation of fluid particle motions. Theor. Comput. Fluid Dyn. 3, 285-306.

Joseph, D. D. \& Ocando, D. 2002 Slip velocity and lift. J. Fluid Mech. 454, 263-286.

Karnis, A., Goldsmith, H. L. \& Mason, S. G. 1966 The flow of suspensions through tubes. Part 5: Inertial effects. Can. J. Chem. Engng 44, 181.

Mclaughlin, J. B. 1991 Inertial migration of a small sphere in linear shear flows. J. Fluid Mech. 224, 261-274.

Matas, J. P., Morris, J. F. \& Guazzelli, E. 2004 Lateral forces on a sphere. Oil Gas Sci. Technol. Rev. 59, 59-70.

Patankar, N. A., Huang, P. Y., Ko, T. \& Joseph, D. D. 2001 a Lift-off of a single particle in Newtonian and viscoelastic fluids by direct numerical simulation. J. Fluid Mech. 438, 67-100.

Patankar, N. A., Ko, T., Choi, H. G. \& Joseph, D. D. $2001 b$ A correlation for the lift-off of many particles in plane Poiseuille of Newtonian fluids. J. Fluid Mech. 445, 55-76.

Rubinow, S. I. \& Keller, J. B. 1961 The transverse force on a spinning sphere moving in a viscous fluid. J. Fluid Mech. 11, 447-459.

SAffman, P. G. 1965 The lift on a small sphere in a slow shear flow. J. Fluid Mech. 22, 385-400; and Corrigendum, 1968 J. Fluid Mech. 31, 624.

Schonberg, J. A. \& Hinch, E. J. 1989 Inertial migration of a sphere in Poiseuille flow. J. Fluid Mech. 203, 517-524.

Segré, G. \& Silberberg, A. 1961 Radial Poiseuille flow of suspensions. Nature, 189, 209.

Segré, G. \& Silberberg, A. 1962 Behaviour of macroscopic rigid spheres in Poiseuille flow. Part 1. J. Fluid Mech. 14, 136-157.

Singh, P., Joseph, D. D., Hesla, T. I., Glowinski, R. \& Pan, T.-W. 2000 A distributed Lagrange multiplier/fictitious domain method for viscoelastic particulate flows. J. Non-Newtonian Fluid Mech. 91, 165-188.

WANG, J. \& JoSEPH, D. D. 2003 Lift forces on a cylindrical particle in plane Poiseuille flow of shear thinning fluids. Phys. Fluids 15, 2267-2278.

ZHU, M.-Y. 2000 Direct numerical simulation of the solid-liquid flows of Newtonian and viscoelastic fluids. PhD thesis, University of Pennsylvania. 